\title{
Cross-cultural differences in language markers of depression online
}

\author{
Kate Loveys \\ Qntfy \\ katedgntfy.com
}

\author{
Jonathan Torrez \\ Qntfy \\ jonathan.torrezegntfy.com
}

\author{
Alex Fine \\ Qntfy \\ alex.finedgntfy.com
}

\author{
Glen Moriarty \\ 7 Cups of Tea \\ glen.moriarty@ 7cups.com
}

\author{
Glen Coppersmith \\ Qntfy \\ glenequt fy.com
}

\begin{abstract}
Depression is a global mental health condition that affects all cultures. Despite this, the way depression is expressed varies by culture. Uptake of machine learning technology for diagnosing mental health conditions means that increasingly more depression classifiers are created from online language data. Yet, culture is rarely considered as a factor affecting online language in this literature. This study explores cultural differences in online language data of users with depression. Written language data from 1,593 users with self-reported depression from the online peer support community 7 Cups of Tea was analyzed using the Linguistic Inquiry and Word Count (LIWC), topic modeling, data visualization, and other techniques. We compared the language of users identifying as White, Black or African American, Hispanic or Latino, and Asian or Pacific Islander. Exploratory analyses revealed crosscultural differences in depression expression in online language data, particularly in relation to emotion expression, cognition, and functioning. The results have important implications for avoiding depression misclassification from machine-driven assessments when used in a clinical setting, and for avoiding inadvertent cultural biases in this line of research more broadly.
\end{abstract}

\section{Introduction}

Depression is a common mental health condition that affects more than 300 million people globally (World Health Organization, 2017). A major contributor to the overall global burden of disease, Major Depression was indicated as the second leading cause of years lived with disability in 2013 (Vos et al., 2015). While effective treatments for depression exist, less than half of those affected by the condition will receive treatment (World Health Organization, 2017). Barriers to appropriate treatment include social stigma associated with mental illness, a lack of resources or trained healthcare providers, and inaccurate assessments (World Health Organization, 2017).

One cause of inaccurate assessment is the use of culturally-inappropriate or -insensitive diagnostic tools; that is, administering an assessment in a cultural context that differs from that in which it was developed, without adaptation or validation (Ng et al., 2016). Inaccurate assessments increase risk of depression misdiagnosis, resulting in patients receiving either incorrect treatment or no treatment at all; both of which may be dangerous outcomes for the patient.

\subsection{Cross-cultural differences in depression experience and expression}

According to some evolutionary psychological approaches to depression, depression is a breakdown in an evolved and adaptive response to experiencing scarcity and loss, particularly in relation to goal attainment, social relationships or status (Nesse and Ellsworth, 2009; Kirmayer et al., 2001); thus, depression is likely to constitute part of the human condition, in some sense independent of culture. Across cultural contexts, depression onset is reliably related to vulnerability factors such as lack of social support, stress, unemployment and poverty, a demanding climate, family history of depression, adverse childhood experiences, and a high level of trait neuroticism (Chentsova-Dutton and Tsai, 2009; Kirmayer et al., 2001; Sullivan et al., 2000; Chapman et al., 2004). While depression affects humans crossculturally, cultural context nevertheless impacts the way depression is experienced and expressed, and plays a role in shaping a community's general beliefs about mental health and illness, and how treatment is approached (Chentsova-Dutton and Tsai, 2009; Ng et al., 2016). 


\subsubsection{Depression expression}

Chentsova-Dutton and Tsai (2009) suggest that cultural scripts about normative and deviant behavior impact expression of depression across cultural contexts. Deviant scripts, specifically those that pertain to depression and the expression of distress, vary amongst cultures. This means that depression symptoms attended to and reported cross-culturally may vary (due to differences in what 'healthy' and 'depressed' functioning mean in context, as well as cultural differences in what are socially acceptable symptoms to report (Kirmayer et al., 2001)).

A large body of literature suggests that cultural differences in depression symptom reporting are reliably observed. However, how these differences in symptom reporting are manifested varies between studies. This is the result of variation in measurement methods (e.g., use of closed versus open-ended self-report questions to evaluate symptoms), degree of acculturation, and other socio-demographic factors at play, such as the socioeconomic status or education level of participants.

Some previous work has found cultural variation in somatic versus psychological symptom reporting for individuals with depression. Due to a prevailing (implicit or explicit) belief in mindbody dualism (Ayalon and Young, 2003) in western cultures, there is a tendency in western cultures to "psychologize" the symptoms of depression, focusing on reporting psychological symptoms (e.g., low mood, cognitive symptoms such as thoughts of hopelessness or excessive guilt) while discussing depression spontaneously (Ryder et al., 2008). Cultures that have traditionally viewed physical and mental health as an interlinked concept, by contrast, might be more likely to spontaneously report somatic symptoms to indicate psychological distress (Ryder et al., 2008), particularly in contexts where mental illness is heavily stigmatized and thus, reporting of somatic symptoms is more socially acceptable (Kirmayer et al., 2001), or somatic symptoms are more heavily embedded at the forefront of the culture's 'script' for depression (Chentsova-Dutton and Tsai, 2009). Somatization tendencies in depression symptom reporting have been observed especially in Asian and Middle Eastern cultures (Chan et al., 2004; Ayalon and Young, 2003).

However, methodology for assessing depres- sion symptoms can impact the degree of somatization observed between cultures (Chan et al., 2004). For example, Ryder et al. (2008) observed that Chinese individuals with depression were more likely to self-report somatic symptoms spontaneously in response to an open-ended question about depression symptoms. When asked closedended questions about depression symptoms in a structured interview, the rate at which Chinese individuals reported experiencing psychological symptoms (e.g., low mood) increased.

Other literature has highlighted how cultural variation in emotion expression norms and ideals impacts how individuals with depression might express or regulate their low mood. One study in particular compared European American and Asian American individuals with depression to non-depressed controls on type and degree of emotion expression following exposure to a sad film (Chentsova-Dutton et al., 2007). Differences in cultural norms pertaining to emotion expression and regulation meant that participants with depression either expressed or regulated sadness in response to the film dependent on culture. In both cases, emotion expression or regulation was opposite to the non-depressed cultural norm. Thus, whether low mood is more likely to be expressed or regulated by individuals with depression varies by culture.

Membership in an individualist or collectivist culture may also have implications for how depression symptoms are reported. Individualism and collectivism can influence the perceived causes of mental health diagnoses, the way conditions are conceptualized, and what is viewed to be an appropriate treatment response (Hall et al., 1999). Members of individualist western cultures tend to view depression as a mental health challenge experienced by the individual, caused by factors related to the individual specifically, and appropriately treated at the individual level. Conversely, collectivist cultures are more likely to conceptualize depression as a family, community, or tribal problem best treated with group involvement and consideration of social factors, with social factors a key contributor to the cause of illness. This may have implications for the ways in which individuals understand and thus talk about their depression. 


\subsubsection{Beliefs about mental illness}

Beliefs about the social acceptability and causes of mental illness can vary across cultural groups and impact how these groups talk about depression, whether members of the group are likely to seek help, and whether depression symptoms are considered to be a medical problem requiring treatment at all (Patel et al., 2016; Aggarwal et al., 2014; Saraceno et al., 2007). In some contexts, depression symptoms are viewed as a normal response to the conditions of human life (ChentsovaDutton and Tsai, 2009), or are perceived to be a 'western' problem (Patel et al., 2016). Eastern Europeans tend to view mild depression symptoms and negative emotion as part of normal functioning (Jurcik et al., 2013; Turvey et al., 2012).

Given the clear evidence for cross-cultural differences in depression experience, expression, and beliefs about mental illness in the clinical literature discussed above, it follows that the ways in which people discuss their depression symptoms online might also vary according to culture.

\subsection{Language markers of depression online}

Many studies have found linguistic predictors of depression in social media and online data more generally. In comparison to healthy controls, depressed individuals tend to write online with greater self-focus (Coppersmith et al., 2014; Preoţiuc-Pietro et al., 2015), tentativeness (Coppersmith et al., 2015), general negativity (De Choudhury et al., 2013), sadness (De Choudhury et al., 2013; Schwartz et al., 2014; PreoţiucPietro et al., 2015), anxiety (Coppersmith et al., 2014), anger (Coppersmith et al., 2014), interpersonal hostility (Preoţiuc-Pietro et al., 2015), swearing (Resnik et al., 2015a), and are more likely to display evidence of anhedonia (PreoţiucPietro et al., 2015), social problems (Schwartz et al., 2014; Resnik et al., 2015a,b), health and sleep issues (Schwartz et al., 2014; Resnik et al., 2015b), inactivity (Coppersmith et al., 2015), death interest (Coppersmith et al., 2015; Preoţiuc-Pietro et al., 2015), perceived hopelessness (Schwartz et al., 2014), and problems in key life domains such as work or school (De Choudhury et al., 2013; Resnik et al., 2015b). Depressed individuals are less likely to discuss leisure (Coppersmith et al., 2015), self-care or exercise (Resnik et al., 2015b), are less likely to provide evidence of engagement in social activities (Resnik et al., 2015a), and are less likely to exhibit positivity in their online language (Resnik et al., 2015a; Reece et al., 2017).

In the studies cited above, individuals were indicated to have depression based on self-reported diagnosis or electronic medical records, and language samples were taken from a diverse set of social media sites and forums, such as Facebook, Twitter, and Reddit. These findings suggest that individuals with depression have quantifiable differences in their use of language online, compared to the general population. However-and crucial to the goals of the current study-it is important to note that most research in this area has been either based on data taken from predominantly Caucasian western populations, or the cultural composition of the samples were simply not reported or analyzed. Thus, a major question in this literature is whether linguistic correlates of depression from internet data hold across different cultural groups.

\subsubsection{Cultural differences in online language markers of depression}

Only one study has examined cultural differences in internet-derived linguistic markers of depression to date. De Choudhury et al. (2017) analyzed Tweets of users who self-reported a diagnosis of depression, 'mental illness', or experiencing suicidal ideation in aggregate. Comparisons were made between 'Western' (United States, United Kingdom) and 'Non-Western' (South Africa, India) groups with the Linguistic Inquiry and Word Count (LIWC2015) software and topic modeling. 'Non-western' cultural groups were more likely to inhibit expression of their mental illness experience online, which manifested in multiple ways: 1) Firstly, 'non-western' individuals with depression expressed higher positive affect and lower negative affect, anger, anxiety, and sadness in comparison to 'western' cultural groups.

2) Secondly, individuals from 'non-western' cultural groups displayed lower cognitive impairment, as evidenced through greater mentions of cognitive processes (e.g. cause, know, ought), certainty terms (e.g. always, never), discrepancies (e.g. should, would), and perceptions (e.g. look, heard, feeling) in comparison to 'western' cultural groups.

3) Additionally, 'western' groups were more likely to discuss functioning, such as social concerns, health, body, and biology, than 'nonwestern' groups. 'Non-western' groups were less 
likely to discuss 'taboo' topics such as religion, death, and sexuality. Topic modeling further revealed cultural differences. 'Western' cultures were more likely to discuss social isolation, death and self-destruction, whereas 'non-western' cultures were more likely to discuss shame from experiencing a mental illness, and make confessions related to their mental health struggles.

These findings suggest that 'non-western' cultural groups tend to inhibit expression of mental illness in online language. In contrast, 'western' groups let the cognitive, emotional, and social experiences of their mental illness be more clearly evident in online language. However, given the nascence of this research, further research is needed to replicate these findings as well as to examine language differences amongst more diverse cultural groups.

\subsection{The present study}

In the current study, we present an exploratory analysis of differences in the linguistic expression of depression across cultural groups within the United States. Specifically, we explore how the language of White, Asian or Pacific Islander, Black or African American, and Hispanic or Latino individuals with depression compared while discussing their mental health on an online mental health support forum.

\section{Methods}

\subsection{Data collection}

Data was collected from 7 Cups of Tea, an anonymous online, chat-based peer support community for emotional distress ${ }^{1}$. Users agree at signup that their data may be used for the purposes of research. All the data used for the current study was anonymous and securely stored. This research was performed in line with the ethical and privacy protocols outlined in detail in (Benton et al., 2017).

Data from 7 Cups takes the form of written dialogue between users of the service and volunteers who are trained as "active listeners". A fragment of an exchange between the user of the service (U) and the volunteer $(\mathbf{V})$ might go as follows:

$$
\begin{aligned}
& \text { V: hey, hows it going } \\
& \text { U: not so good } \\
& \text { V: wanna tell me about it? }
\end{aligned}
$$

For the analyses reported in this paper, we used only text generated by users of the service, not the volunteers providing peer support.

Users who reported depression as their primary concern at sign up were eligible for inclusion in analyses. Our original sample was comprised of 23,048 conversations involving 1,937 unique users. Users were excluded from the sample if they did not indicate their culture, or if they selected 'Other'. This resulted in the exclusion of 199 and 130 users, respectively. The original sample also included users identifying as Native American or American Indian. This group was excluded from analyses since the majority of the data among these users was not English. This resulted in the removal of 15 users, leaving a total sample size of 1,593 .

\subsection{Measures}

Users of the service completed a questionnaire at sign-up in which they provided information about their demographic characteristics and mental health. Demographic characteristics assessed included age, gender, and ethnicity. Ethnicity response categories were White, Asian or Pacific Islander, Black or African American, Latino or Hispanic, Native American or American Indian, or Other. Users could only select one ethnic group category. Users also select the primary reason for using 7 Cups, and the users above all indicated a primary purpose of "Depression".

\section{Results}

We report descriptive statistics of the sample, LIWC analyses, and the results of a topic modeling analysis.

\subsection{Descriptive statistics}

Data was anonymous and users were analyzed in aggregate by cultural group. No personallyidentifiable information was available. We report descriptive statistics to give a sense of the overall composition of the sample.

Table 1 outlines demographic characteristics and mental health status of participants. Overall, participants were predominantly female $(67.3$ percent), white (68.6 percent), young adults ( $\mathrm{m}=$ 21.4, $\mathrm{SD}=7.6$ ), who were somewhat distressed at sign-up (7/10). No statistically significant or meaningful differences in age, gender, or sign-up distress level were found between cultural groups 
and thus, these characteristics were not controlled for in subsequent analyses.

\subsection{LIWC analyses}

Next, exploratory analyses were conducted with the Linguistic Inquiry and Word Count software (LIWC2015) (Pennebaker et al., 2015), which is a psychometrically-validated program that evaluates the percentage of total words in a document that relate to different psychological constructs (e.g., "emotion", "cognition") or life domains (e.g., "health", "social"). LIWC has been used in prior research evaluating social media language patterns of diverse samples with depression (Coppersmith et al., 2015; De Choudhury et al., 2017). LIWC analyses in the present study compared language of White, Asian or Pacific Islander, Black or African American, and Hispanic or Latino users with depression.

Language analyses with LIWC were exploratory in nature and thus we compared cultural groups on the degree to which they expressed content about a wide range of relevant topics, including emotion, cognitive impairment, social functioning, health, and taboo topics. Given the large amount of language comparisons made between cultural groups, we draw the reader's attention to several interesting findings in light of existing cross-cultural depression literature (see Figure 1). Note also that due to the exploratory nature of the current study, we do not conduct or report statistical tests over the LIWC results. In the absence of a specific hypothesis about the distribution over LIWC scores, conditioned on ethnic group and LIWC category, statistical tests such as ANOVA would be misleading at best. We hope that the current exploratory analyses will guide future hypothesis-driven work.

First, cultural differences in degree and type of emotion expression were observed. Here, emotion is captured by the LIWC category "tone", which reprsents the ratio of positive to negative emotion expression. Asian or Pacific Islander users showed more inhibition of negative emotion, whereas White and Black or African American users expressed more negativity (in other words, exhibited less regulation of their negative emotional state). Hispanic or Latino users expressed a large amount of both positive and negative emotion compared to other groups.

Second, cultural differences in cognitive cate- gories were observed, whereby cognitive effects of depression were less evident in language of Asian or Pacific Islander users.

Third, discussions of functioning were impacted by culture. White users appeared less social, and were more likely to report on health and death or self-destruction compared to other groups. Asian or Pacific Islander users were less open to discussing health or death, though social terms were more present. Black or African American users discussed social terms to a high degree, and were comparatively less likely to discuss death, but were more willing to talk about health compared to other groups. Opposite to Black or African American users, Hispanic or Latino users with depression had low mentions of social terms and were less willing to make disclosures about death or self-destruction, religion, or health.

Our findings suggest that different cultural groups may be more or less willing to spontaneously discuss particular topics relevant to mental health online. This may have implications when looking to detect individuals with depression from online data, particularly when the sample population is culturally diverse.

\subsection{Topic modeling}

Users' messages were analyzed with topic modeling to provide qualitative assessments of the emergent topics or themes that users wished to discuss with volunteers on the platform. While topic modeling may miss some of the fine-grained insights into users' concerns that a human observer could provide, because it is an unsupervised, datadriven approach to analyzing linguistic data, it offers the intriguing possibility of discovering patterns in users' preoccupations that a human observer would be less likely to identify.

Topics were obtained by running Latent Dirichlet Allocation (LDA) over each cultural group's messages, i.e. one topic model was created per cultural group and an individual document in each corpus was a single user message. The data was pre-processed by removing chat-specific stopwords, words with very high frequency (occurring in more than $75 \%$ of the documents) and words that occur fewer than five times. We then used Gensim's implementation of multi-core LDA with the default hyper-parameter settings and three topics.

Analysis of the terms that were assigned to top- 


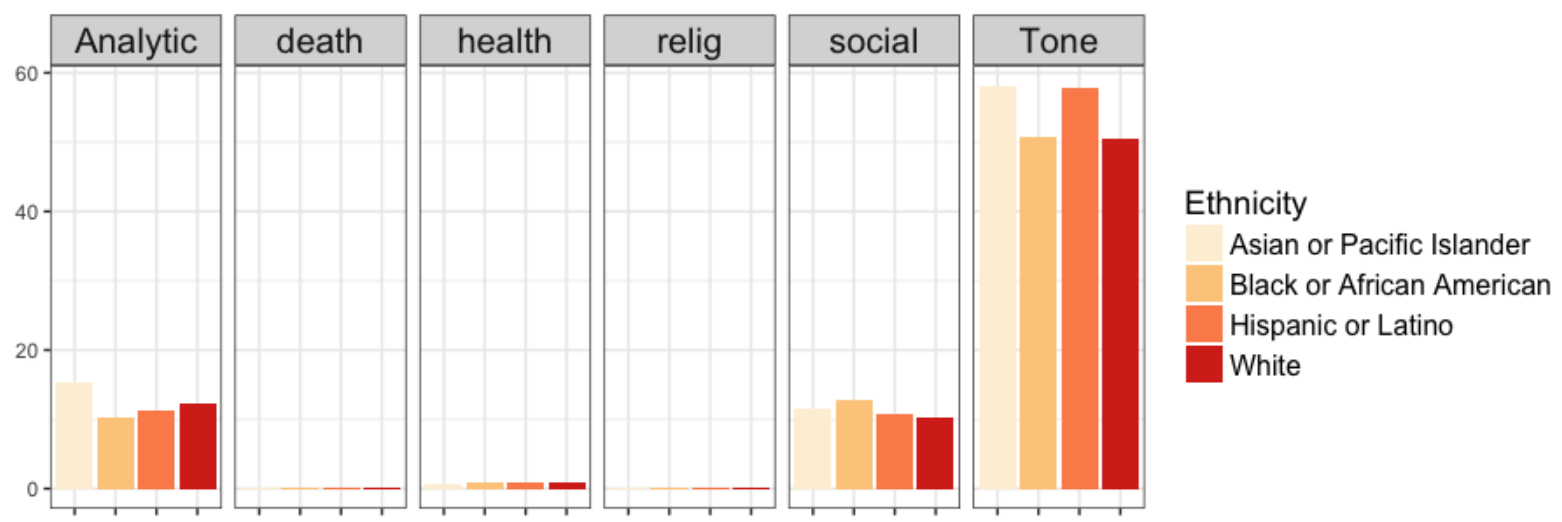

Figure 1: LIWC scores across cultural groups for select themes.
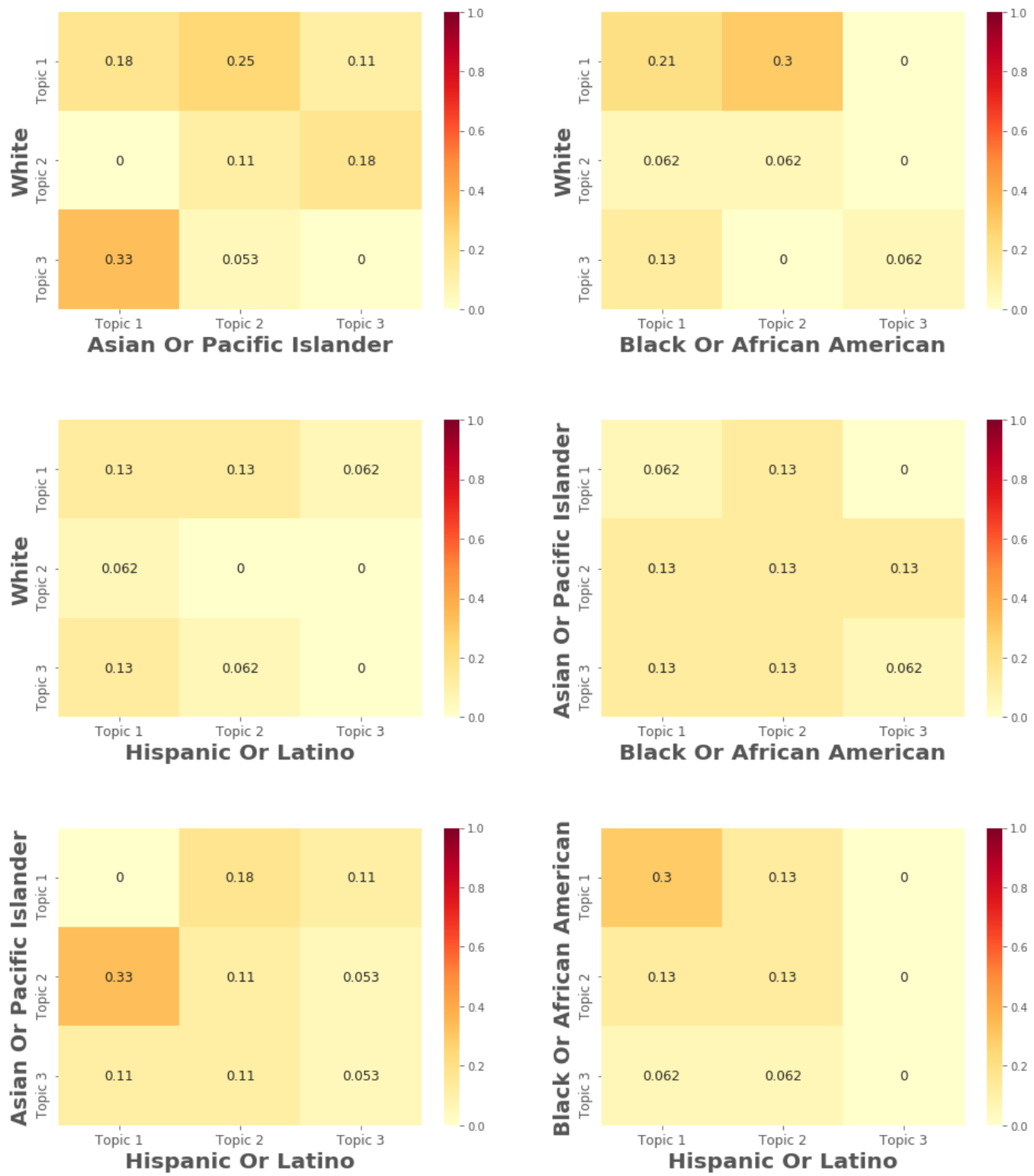

Figure 2: Jaccard similarity coefficients of topics across cultural groups. Darker shades indicate stronger similarities between topics. 


\begin{tabular}{llllll}
\hline & Total & White & Asian or Pac. Islander & Black or Afr. American & Hisp. or Latino \\
\hline Total N (N, \%) & 1,593 & $1,093(68.6)$ & $280(17.6)$ & $92(5.8)$ & $128(8.0)$ \\
& & & Demographic Variables & \\
\hline Age (M, SD) & $21.4(7.6)$ & $21.7(8.4)$ & $21.2(5.4)$ & $22.0(7.0)$ & $19.7(5.3)$ \\
$\begin{array}{l}\text { Gender (\%) } \\
\text { Female }\end{array}$ & 67.3 & 65.3 & 66.4 & 78.3 & 78.1 \\
$\begin{array}{l}\text { Male } \\
\text { Other }\end{array}$ & 29.2 & 30.6 & 31.1 & 20.0 & 2.0 \\
& 3.5 & 4.0 & 2.5 & 2.2 & \\
\hline Sign-up Distress (M, SD) & $6.9(1.9)$ & $6.9(1.9)$ & $7.1(1.8)$ & $6.8(1.8)$ & $6.4(2.1)$ \\
\hline
\end{tabular}

Table 1: Descriptive statistics of sample demographics and mental health

\begin{tabular}{|l|l|l|l|}
\hline Cultural Group & Topic 1 & Topic 2 & Topic 3 \\
\hline Asian \& Pacific Islander & $\begin{array}{l}\text { work need bad } \\
\text { sorry girl }\end{array}$ & $\begin{array}{l}\text { friend love feeling } \\
\text { maybe guy }\end{array}$ & $\begin{array}{l}\text { year friend can't } \\
\text { care actually }\end{array}$ \\
\hline Black or African American & $\begin{array}{l}\text { friend feeling work } \\
\text { help hard }\end{array}$ & $\begin{array}{l}\text { friend need year } \\
\text { car care }\end{array}$ & $\begin{array}{l}\text { work can't better } \\
\text { trying mom }\end{array}$ \\
\hline Hispanic or Latino & $\begin{array}{l}\text { friend can't year } \\
\text { feeling hope }\end{array}$ & $\begin{array}{l}\text { need bad help bet- } \\
\text { ter sad }\end{array}$ & $\begin{array}{l}\text { work nice love de- } \\
\text { pression long }\end{array}$ \\
\hline White & $\begin{array}{l}\text { need love friend } \\
\text { help bad }\end{array}$ & $\begin{array}{l}\text { year can't hate } \\
\text { come look }\end{array}$ & $\begin{array}{l}\text { sorry work didn't } \\
\text { better live }\end{array}$ \\
\hline
\end{tabular}

Table 2: Top five terms per topic across cultural groups. Italicized terms appear in top five terms for all groups. Bolded terms only appear in the top five terms list for one cultural group.

ics per cultural group revealed that among the top three topics for each cultural group, there was little overlap in terms. Term overlap was measured using the Jaccard similarity coefficient and is shown in Fig. 2. The similarity coeffecient can be interpreted as the percent overlap of the set of terms in each topic. A similarity coefficient of 1 would indicate that all terms assigned to two different topics were exactly the same. Values higher than 0.3 , indicating approximately a third of terms were shared in common between two topics, occurred only four times out of fifty-four topic comparisons. Most coefficients are closer to 0.1 and there are many topics with no term overlap. Further work involving analysis of term overlap among members of the same cultural group and computing the difference in topic distribution between groups (by comparing to a single overall topic model) would further illuminate what topical diffences there are between cultural groups.

Further analysis of the specific terms assigned to each topic is captured in Table 2, which shows the top five terms associated with each topic across cultural groups. These sorts of visualizations often resist neat, intuitive explanations. The collection of terms in each topic do not seem to form cohesive topics (e.g., emotions, relationships, etc.), and specific terms (e.g., 'work', 'friend', and 'need') appear across multiple topics, both within a single cultural group and across cultural groups. Topics discussed by all groups may be relevant to individuals with depression cross-culturally; for example, analyses revealed all cultural groups made disclosures about the topic, 'friend', which suggests loneliness or 'need of a friend' is a concern for individuals with depression that cuts across culture. However, the collection of terms in each topic does vary across cultural groups, indicating that there are differences in the themes discussed by users belonging to different cultures. Further work is likely to involve mapping the original chat messages to the topics they are most likely to belong to in order to extract human-interpretable descriptions of the different topics.

\section{Discussion}

Our overall conclusion is consistent with existing cross-cultural depression research (De Choudhury et al., 2017), namely: there are cross-cultural differences in online language of individuals with 
depression. Our results highlight the importance of creating culturally-adapted depression classifiers as automated assessments become increasingly commonplace in the treatment and identification of mental health issue, and suggest a role for research of this kind in developing culturally sensitive clinical instruments for measuring depression.

Our study included a broad range of cultural groups analyzed relative to previous work. Moreover, our use of both closed- and open-vocabulary analyses allowed for both theory-informed and data-driven analyses of language of many diverse cultural groups with depression, which similarly complements the currently existing body of literature on this subject.

A few caveats are worth noting. First, the data reported here was taken from a peer support community specifically for providing emotional support. It is therefore unclear whether and to what extent our findings generalize to other online spaces such as social media, where discussions about mental health are not explicitly encouraged, and where anonymity is not guaranteed. Some previous work has identified cross-cultural differences in language about mental health on social media, albeit for different cultural groups to the present study (De Choudhury et al., 2017). This suggests that our results are unlikely to reflect idiosyncratic properties of the platform, though a rigorous examination of this question must be left to future work.

Second, data about participants' country of residence and extent of acculturation were missing. Thus, the extent to which users were acculturated to western beliefs about mental illness or held traditional mental health beliefs of their culture is somewhat unclear. There is evidence to suggest acculturated individuals report symptoms differently compared to individuals more entrenched in the health beliefs that prevail in their culture (Jang et al., 2005). For example, it was not clear from the available data whether an individual identifying as 'white' was a white American or, for instance, a white German living in the United States. This is relevant since it is reasonable to suppose that white Americans and white Germans are not identical to each other in the way they think and talk about mental health. Similarly, it is plausible that a third-generation Korean American, on the one hand, and a Korean citizen living in the
United States, on the other, would both identify as "Asian American", though it would be odd to classify these individuals as having the same culture for the purposes of the current analysis. The relative frequency of these types of observations is unknown.

A third limitation of this study was the labels used to define groups in our dataset, which include a mix of ethnic and racial groups. These labels were determined by the peer support community. While our cultural group labels were imperfect, we were still able to observe meaningful differences between groups, as well as to conduct a more fine-grained cultural analysis comparative to prior literature in this area, which compared 'Western' to 'non-Western' cultures (De Choudhury et al., 2017).

This paper adds to a small but growing literature examining cross-cultural differences in the way symptoms of depression are expressed in online language data. Our findings have important implications for designing automated depression assessments with online data, and suggest that making good predictions about mental health on the basis of language data will require taking cultural/ethnic identity into account. Should machine-driven depression assessments be deployed in a clinical setting, culturally sensitive classifiers may be necessary to avoid misdiagnosis, a key barrier to receiving effective treatment for depression (World Health Organization, 2017). In future and ongoing work, we plan to extend these analyses to mental health conditions apart from depression, or to focus on depression subtypes, and to deepen this approach by using our exploratory analyses as a springboard for hypothesis-driven work oriented towards informing mental health-related interventions and mental health policy.

\section{Conclusion}

In conclusion, findings from this exploratory study suggest there are cultural differences in online language of individuals with depression. Differences found in the degree to which culturally-diverse individuals with depression express particular topics relevant to mental health online suggest careful attention is required to the cultural contexts in which language classifiers for depression are deployed. Appropriate adaptations, such as depression classifiers made for the cultural group of in- 
terest, may be necessary to avoid misclassification and thus, inappropriate treatment responses. Moreover, these findings suggest a path forward for empirically-driven assessment and creation of cultural sensitivity best practices for online therapy and peer support, based on the concerns and experiences of the people seeking help.

\section{Acknowledgements}

The authors would like to thank the members of the 7 Cups of Tea community for their participation in this research and analysis. Without their participation, this research would not be possible.

\section{References}

Neil Krishan Aggarwal, Madhumitha Balaji, Shuba Kumar, Rani Mohanraj, Atif Rahman, Helena Verdeli, Ricardo Araya, MJD Jordans, Neerja Chowdhary, and Vikram Patel. 2014. Using consumer perspectives to inform the cultural adaptation of psychological treatments for depression: a mixed methods study from South Asia. Journal of affective disorders, 163:88-101.

Liat Ayalon and Michael A Young. 2003. A comparison of depressive symptons in African Americans and Caucasian Americans. Journal of CrossCultural Psychology, 34(1):111-124.

Adrian Benton, Glen Coppersmith, and Mark Dredze. 2017. Ethical research protocols for social media health research. In Proceedings of the First ACL Workshop on Ethics in Natural Language Processing, pages 94-102.

Bibiana Chan, Gordon Parker, Bibiana Chan, and Gordon Parker. 2004. Some recommendations to assess depression in Chinese people in Australasia. Australian \& New Zealand Journal of Psychiatry, 38(3):141-147.

Daniel P Chapman, Charles L Whitfield, Vincent J Felitti, Shanta R Dube, Valerie J Edwards, and Robert F Anda. 2004. Adverse childhood experiences and the risk of depressive disorders in adulthood. Journal of affective disorders, 82(2):217-225.

Yulia E Chentsova-Dutton, Joyce P Chu, Jeanne L Tsai, Jonathan Rottenberg, James J Gross, and Ian H Gotlib. 2007. Depression and emotional reactivity: variation among Asian Americans of East Asian descent and European Americans. Journal of abnormal psychology, 116(4):776.

Yulia E Chentsova-Dutton and Jeanne L Tsai. 2009. Understanding depression across cultures. Handbook of depression, 2:363-385.

Glen Coppersmith, Mark Dredze, and Craig Harman. 2014. Quantifying mental health signals in Twitter.
In Proceedings of the Workshop on Computational Linguistics and Clinical Psychology: From Linguistic Signal to Clinical Reality, pages 51-60.

Glen Coppersmith, Mark Dredze, Craig Harman, and Kristy Hollingshead. 2015. From ADHD to SAD: Analyzing the language of mental health on Twitter through self-reported diagnoses. In Proceedings of the 2nd Workshop on Computational Linguistics and Clinical Psychology: From Linguistic Signal to Clinical Reality, pages 1-10.

Munmun De Choudhury, Michael Gamon, Scott Counts, and Eric Horvitz. 2013. Predicting depression via social media. ICWSM, 13:1-10.

Munmun De Choudhury, Sanket S Sharma, Tomaz Logar, Wouter Eekhout, and René Clausen Nielsen. 2017. Gender and cross-cultural differences in social media disclosures of mental illness. In Proceedings of the 2017 ACM Conference on Computer Supported Cooperative Work and Social Computing, pages 353-369. ACM.

Gordon C Nagayama Hall, Anita Bansal, and Irene R Lopez. 1999. Ethnicity and psychopathology: A meta-analytic review of 31 years of comparative MMPI/MMPI-2 research. Psychological assessment, 11(2):186.

Y Jang, G Kim, and D Chiriboga. 2005. Acculturation and manifestation of depressive symptoms among Korean-American older adults. Aging \& Mental Health, 9(6):500-507.

Tomas Jurcik, Yulia E Chentsova-Dutton, Ielyzaveta Solopieieva-Jurcikova, and Andrew G Ryder. 2013. Russians in treatment: The evidence base supporting cultural adaptations. Journal of clinical psychology, 69(7):774-791.

Laurence J Kirmayer et al. 2001. Cultural variations in the clinical presentation of depression and anxiety: implications for diagnosis and treatment. Journal of Clinical Psychiatry, 62:22-30.

Randolph M Nesse and Phoebe C Ellsworth. 2009. Evolution, emotions, and emotional disorders. American Psychologist, 64(2):129.

Lauren C Ng, Jessica F Magidson, Rebecca S Hock, John A Joska, Abebaw Fekadu, Charlotte Hanlon, Janina R Galler, Steven A Safren, Christina PC Borba, Gregory L Fricchione, et al. 2016. Proposed training areas for global mental health researchers. Academic Psychiatry, 40(4):679-685.

Vikram Patel, Dan Chisholm, Rachana Parikh, Fiona J Charlson, Louisa Degenhardt, Tarun Dua, Alize J Ferrari, Steve Hyman, Ramanan Laxminarayan, Carol Levin, et al. 2016. Addressing the burden of mental, neurological, and substance use disorders: key messages from disease control priorities. The Lancet, 387(10028):1672-1685. 
James W Pennebaker, Ryan L Boyd, Kayla Jordan, and Kate Blackburn. 2015. The development and psychometric properties of LIWC2015. Technical report.

Daniel Preotiuc-Pietro, Johannes Eichstaedt, Gregory Park, Maarten Sap, Laura Smith, Victoria Tobolsky, H Andrew Schwartz, and Lyle Ungar. 2015. The role of personality, age, and gender in tweeting about mental illness. In Proceedings of the 2 nd Workshop on Computational Linguistics and Clinical Psychology: From Linguistic Signal to Clinical Reality, pages 21-30.

Andrew G Reece, Andrew J Reagan, Katharina LM Lix, Peter Sheridan Dodds, Christopher M Danforth, and Ellen J Langer. 2017. Forecasting the onset and course of mental illness with Twitter data. Scientific reports, 7(1):13006.

Philip Resnik, William Armstrong, Leonardo Claudino, and Thang Nguyen. 2015a. The University of Maryland CLPsych 2015 shared task system. In Proceedings of the 2nd Workshop on Computational Linguistics and Clinical Psychology: From Linguistic Signal to Clinical Reality, pages 54-60.

Philip Resnik, William Armstrong, Leonardo Claudino, Thang Nguyen, Viet-An Nguyen, and Jordan Boyd-Graber. 2015b. Beyond LDA: exploring supervised topic modeling for depressionrelated language in Twitter. In Proceedings of the 2nd Workshop on Computational Linguistics and Clinical Psychology: From Linguistic Signal to Clinical Reality, pages 99-107.

Andrew G Ryder, Jian Yang, Xiongzhao Zhu, Shuqiao Yao, Jinyao Yi, Steven J Heine, and R Michael Bagby. 2008. The cultural shaping of depression: somatic symptoms in China, psychological symptoms in North America? Journal of abnormal psychology, 117(2):300.

Benedetto Saraceno, Mark van Ommeren, Rajaie Batniji, Alex Cohen, Oye Gureje, John Mahoney, Devi Sridhar, and Chris Underhill. 2007. Barriers to improvement of mental health services in lowincome and middle-income countries. The Lancet, 370(9593):1164-1174.

H Andrew Schwartz, Johannes Eichstaedt, Margaret L Kern, Gregory Park, Maarten Sap, David Stillwell, Michal Kosinski, and Lyle Ungar. 2014. Towards assessing changes in degree of depression through Facebook. In Proceedings of the Workshop on Computational Linguistics and Clinical Psychology: From Linguistic Signal to Clinical Reality, pages 118-125.

Patrick F Sullivan, Michael C Neale, and Kenneth S Kendler. 2000. Genetic epidemiology of major depression: review and meta-analysis. American Journal of Psychiatry, 157(10):1552-1562.
Carolyn L Turvey, Gerald Jogerst, Mee Young Kim, and Elena Frolova. 2012. Cultural differences in depression-related stigma in late-life: a comparison between the usa, russia, and south korea. International psychogeriatrics, 24(10):1642-1647.

Theo Vos, Ryan M Barber, Brad Bell, Amelia BertozziVilla, Stan Biryukov, Ian Bolliger, Fiona Charlson, Adrian Davis, Louisa Degenhardt, Daniel Dicker, et al. 2015. Global, regional, and national incidence, prevalence, and years lived with disability for 301 acute and chronic diseases and injuries in 188 countries, 1990-2013: a systematic analysis for the global burden of disease study 2013. The Lancet, 386(9995):743-800.

World Health Organization. 2017. Depression fact sheet. 